\title{
Fluoridated milk may be beneficial to schoolchildren by helping prevent caries
}

\author{
Does drinking fluoridated milk help prevent dental caries in children?
}

\begin{abstract}
Yeung CA, Hitchings JL, Macfarlane TV, Threlfall AG, Tickle M, Glenny AM. Fluoridated milk for preventing dental caries. The Cochrane Database of Systematic Reviews 2005, Issue 3. Art. No. CD003876
\end{abstract}

Data sources Data sources were the Cochrane Oral Health Group Trials Register, Cochrane Central Register of Controlled Trials, Medline and OLDMedline, Embase, LILACS (Latin American and Caribbean Health Sciences Literature), BBO (Brazilian Bibliography of Dentistry), SIGLE (System for Information on Grey Literature in Europe) and Digital Dissertations. Bibliographies of relevant articles were searched and attempts were made to identify unpublished and ongoing studies. Several relevant journals were also searched by hand.

Study selection There was no language restriction. Randomised controlled trials (RCT) or quasi-RCT were selected if they had an intervention or follow-up period of $>3$ years and compared fluoridated and nonfluoridated milk.

Data extraction and synthesis Inclusion criteria, data extraction and quality assessment were carried out independently and in duplicate, with disagreements being resolved by discussion and agreement being assessed using Kappa. Mean differences and standard deviations were used to summarise data.

Results Out of 144 articles identified by the searches, only two met the inclusion criteria. Since only one report was available as an abstract, unpublished information was requested from the authors and included in this review. A total of 353 children, who had baseline ages of 3-5.5 years, were involved in the studies. The fluoride levels in the two reports differed $(2.5$ or $7.5 \mathrm{mg} / \mathrm{l})$ and therefore their results could not be combined. After consumption of fluoridated milk for 3 years there was a significant reduction in DMFT $(78.4 \%, P<0.05)$ between test and control groups in one study. In the other study, although mean DMFT and DMFS were more favourable in the test group, no significant reductions in DMFT were observed until the fourth $(35.5 \%, P<0.02)$ and fifth years $(31.2 \%, P<0.05)$. For primary teeth there was a significant reduction in DMFT $(31.3 \%, P<0.05)$ between the test and control groups after 3 years in one study but not the other. No intentto-treat analysis could be carried out.

Conclusions The two studies that were of sufficient quality to be included in this review suggested that fluoridated milk was beneficial to schoolchildren by helping prevent caries in the permanent dentition. There remains, however, a dearth of studies providing high-quality evidence of the effects of fluoridated milk.

\section{Commentary}

Milk is an attractive vehicle for fluoride because it forms an important part of children's diets, having natural caries-protective and health-promoting components. The research question is also highly relevant in the light of the fact that fluoridated milk schemes are in progress in several countries in Asia, South America and Europe.

Address for correspondence: Emma Tavender, Review Group Co-ordinator, Cochrane Oral Health Group, University Dental Hospital of Manchester, Higher Cambridge Street, Manchester M15 6FH, UK. E-mail: emma.tavender@man.ac.uk
The authors evaluated nearly 200 publications using commonly adopted quality criteria for caries trials with the thoroughness and excellence expected from the Cochrane Oral Health Group. Only two papers fulfilled the criteria of study selection and, unfortunately, the results of the two trials could not be combined. Therefore, the conclusion that fluoridated milk may be beneficial to schoolchildren, and especially their permanent dentition, is surprisingly strong.

One of the included studies recruited preschool children at baseline and it is questionable whether an intervention between 3 and 6 years of age permits any firm conclusion on the cariesinhibiting effect in the permanent dentition. The permanent teeth have simply not been erupted for long enough to be decayed and this was reflected by the very low caries prevalence in the test and control groups (mean DMFT, 0.04 vs 0.17). Moreover, at this age, the caries-prone surfaces are the fissures of the first permanent molars: these present diagnostic problems when using visual and tactile methods and thus have a high risk for diagnostic errors.

The second study used milk with a threefold higher fluoride content, but failed to show significant differences compared with nonfluoridated milk until the fourth and the fifth years of the trial: this clearly illustrates that a fluoride milk programme must continue for several years if it is to have an impact on oral health. It should also be noted that the latter study suffered from a large attrition rate, and that the milk was ingested using a straw, which may not be optimal from a caries-preventive point of view.

Since the results of the two trials concerning the primary dentition were somewhat mixed, it was not possible to present evidence for the preventive effect of fluoridated milk on primary teeth. The criteria for intervention or follow-up were at least 3 years, which could be argued to be rather long bearing in mind the rapid progression rate of caries in primary teeth, but a reduction in this case would not have increased the number of RCTs.

The authors make a key point when they state, "although there was little robust evidence to support fluoridated milk and the external validity of the included studies must be viewed with caution, this does not imply fluoridated milk is ineffective in caries prevention, merely that high-quality RCT evidence is lacking in the area". The method is probably good both for general and dental health but scientists have not yet been able to prove it. This should be looked upon as an urgent challenge rather than a problem.

\section{Practice point}

- Available evidence suggests a beneficial effect of fluoridated milk in preventing dental caries. However, high-quality RCTs are needed to provide a definitive answer.

\section{Svante Twetman \\ Department of Odontology, Faculty of Medicine, Umea University, Umeå, Sweden}

Evidence-Based Dentistry (2005) 6, 88.

doi:10.1038/sj.ebd.6400357 